\title{
Feasibility Study of Stochastic Streaming with 4K UHD Video Traces
}

\author{
Joongheon $\mathrm{Kim}^{\dagger}$ and Eun-Seok Ryu ${ }^{\ddagger}$ \\ ${ }^{\dagger}$ Platform Engineering Group, Intel Corporation, Santa Clara, California, USA \\ ${ }^{\ddagger}$ Department of Computer Engineering, Gachon University, Republic of Korea \\ Emails: joongheon@gmail.com ${ }^{\dagger}$, esryu@gachon.ac.kr ${ }^{\ddagger}$
}

\begin{abstract}
This paper performs the feasibility study of stochastic video streaming algorithms with up-to-date $4 \mathrm{~K}$ ultra-highdefinition (UHD) video traces. In previous work, various stochastic video streaming algorithms were proposed which maximize time-average video streaming quality subject to queue stability based on the information of queue-backlog length. The performance improvements with the stochastic video streaming algorithms were verified with traditional MPEG test sequences; but there is no study how much the proposed stochastic algorithm is better when we consider up-to-date 4K UHD video traces. Therefore, this paper evaluates the stochastic streaming algorithms with 4K UHD video traces; and verifies that the stochastic algorithms perform better than queue-independent algorithms, as desired.
\end{abstract}

Index Terms-Stochastic streaming, $4 \mathrm{~K}$ ultra-high-definition (UHD) video, Performance evaluation, Feasibility study

\section{INTRODUCTION}

According to the predictions from the Cisco Visual Networking Index (VNI) [1], the summation of all possible forms of video contents will constitute $80 \%$ to $90 \%$ of global data traffic by 2017, and the traffic from mobile and wireless portable devices will exceed the traffic from wired devices by 2016. Therefore, efficient wireless video streaming algorithms are of the highest importance [2].

Based on this importance, various types of video streaming algorithms have been investigated; and one of major research directions is stochastic video streaming which aiming at the time-average video quality maximization subject to video queue/buffer stability [3]-[8]. In [3]-[5], stochastic video streaming algorithms for device-to-device distributed computing systems are proposed. In [3], device-to-device stochastic video streaming with two types of schedulers (centralized vs. distributed) is discussed; and the related performance evaluation with various settings is performed. In [6]-[8], stochastic video streaming in small cell networks is proposed; and the corresponding theoretical analysis is also presented.

In the two research directions, they discuss about stochastic network optimization applications to adaptive video streaming (i.e., stochastic streaming) which maximizes time-average video streaming quality subject to queue/buffer stability. If we transmit maximum quality video streams all the time, the streaming quality will be maximized whereas the queue/buffer within the transmitter will be overflowed. On the other hand, if we transmit minimum quality video streams all the time, the queue/buffer will be stable whereas the streaming quality will

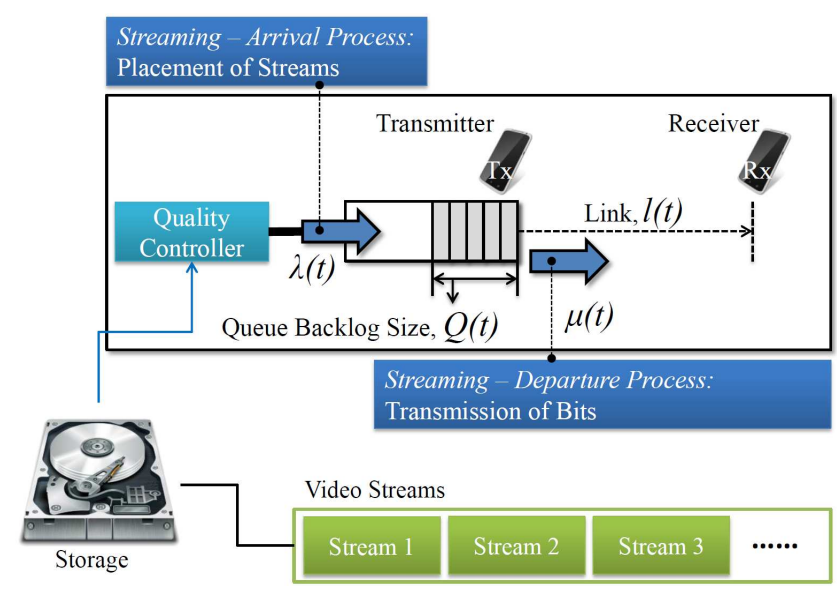

Fig. 1. A stochastic streaming model

be minimized. Therefore, the proposed stochastic streaming adapts the quality of each video stream depending on current queue-backlog length [3]-[8].

In [3]-[8], the used video traces are MPEG test sequences, however the test sequences are not used in current consumer electronics applications. Therefore, this paper evaluates the stochastic streaming algorithms with up-to-date $4 \mathrm{~K}$ ultra-highdefinition (UHD) video test sequences. After observing the performance evaluation results with $4 \mathrm{~K}$ UHD video traces, we can numerically identify how much the novel stochastic streaming algorithm is better than queue-independent nonadaptive video streaming algorithms.

The remainder of this paper is organized as follows: Section II explains the proposed stochastic video streaming algorithm in [3], [4]. Section III shows the simulation results with various simulation parameter settings and with the $4 \mathrm{~K}$ UHD video traces. Section IV concludes this paper and presents future research directions.

\section{Proposed Stochastic Streaming in [3], [4]}

As well-studied in [3], [4] and also shown in Fig. 1, the proposed stochastic video streaming consists of two parts, i.e., (i) placement of streams (i.e., arrival process of the queue/buffer) and (ii) transmission of bits (i.e., departure process of the queue/buffer).

The placement of streams happens in each stream time $t_{s}$ 
and also the transmission of bits happens in each unit time $t$, respectively. It means both unit time and stream time have different time clock as explained in [3]. In this paper, stream placement happens when

$$
t \bmod K=0
$$

where $K$ is a positive integer value which is the scaling factor between stream time and unit time. In addition, stream time can be defined as follows only when $t \bmod K=0$ :

$$
t_{s}=\left\{\begin{array}{cc}
0, & t=0 \\
\frac{t}{K}, & t \neq 0 .
\end{array}\right.
$$

In the given system in Fig. 1, the queue dynamics can be formulated as follows:

$$
Q(t+1)=\max \{Q(t)+\lambda(t)-\mu(t), 0\}
$$

where $t \in\{0,1,2, \cdots\}, Q(t)$ is queue backlog length in unit time $t, \lambda(t)$ is the arrival process of the queue/buffer (i.e., placement of streams and the details are in Section II-1), and $\mu(t)$ is the departure process of the queue/buffer (i.e., transmission of bits and the details are in Section II-2).

1) Arrival Process (Placement of Streams): In each stream time slot $t_{s}$, the transmitter of each link places a stream into its transmission queue. This is the arrival process of the given system, and it is denoted as $\lambda(t)$ in Fig. 1.

In order to dynamically and adaptively select the quality level of the streams by the Quality Controller in Fig. 1, we consider stochastic network optimization frameworks for maximizing the total time-average video quality subject to queue stability.

Then, the proposed stochastic optimization problem is given by:

$$
\begin{array}{cl}
\max & \lim _{t \rightarrow \infty} \frac{1}{t} \sum_{t_{s}=0}^{t-1} \mathbb{E}\left[\mathbb{P}\left(q\left(t_{s}\right), t_{s}\right)\right] \\
\text { subject to } & \lim _{t \rightarrow \infty} \frac{1}{t} \sum_{t_{s}=0}^{t-1} \mathbb{E}\left[Q\left(q\left(t_{s}\right), t_{s}\right)\right]<\infty
\end{array}
$$

where $\mathbb{P}\left(q\left(t_{s}\right), t_{s}\right)$ is the peak-signal-to-noise-ratio (PSNR) of a current stream in stream time $t_{s}$ when the quality mode is $q\left(t_{s}\right), Q\left(q\left(t_{s}\right), t_{s}\right)$ is the queue backlog length in stream time $t_{s}$ when the quality mode is $q\left(t_{s}\right)$, and (5) stands for the given queue should fulfill queue stability [10]. Note that PSNR is one of representative indices for numerically identifying the quality of video frames [11]. In addition, the $\mathbb{P}\left(q\left(t_{s}\right), t_{s}\right)$ and $Q\left(q\left(t_{s}\right), t_{s}\right)$ in Eq. (4) and Eq. (5) can vary depending on quality modes. If the quality mode is for maximum quality, the $\mathbb{P}\left(q\left(t_{s}\right), t_{s}\right)$ and $Q\left(q\left(t_{s}\right), t_{s}\right)$ in Eq. (4) and Eq. (5) will be maximum by assuming that higher quality streams have the large amounts of bits for more detailed representation of video contents.

As theoretically discussed and proved in [3], the quality control decision involves choosing our optimal $q^{*}\left(t_{s}\right)$ for the time-average stochastic optimization framework in Eq. (4) and Eq. (5) as follows:

\section{Parameter setting}

- $K$ : scaling factor between stream time and unit time

- $V$ : tradeoff between video quality and queue stability

- $M$ : set of possible quality modes

- BW: channel bandwidth of the system

- $P^{\mathrm{Tx}}$ : transmit power

- $N_{\mathrm{mW}}$ : background noise

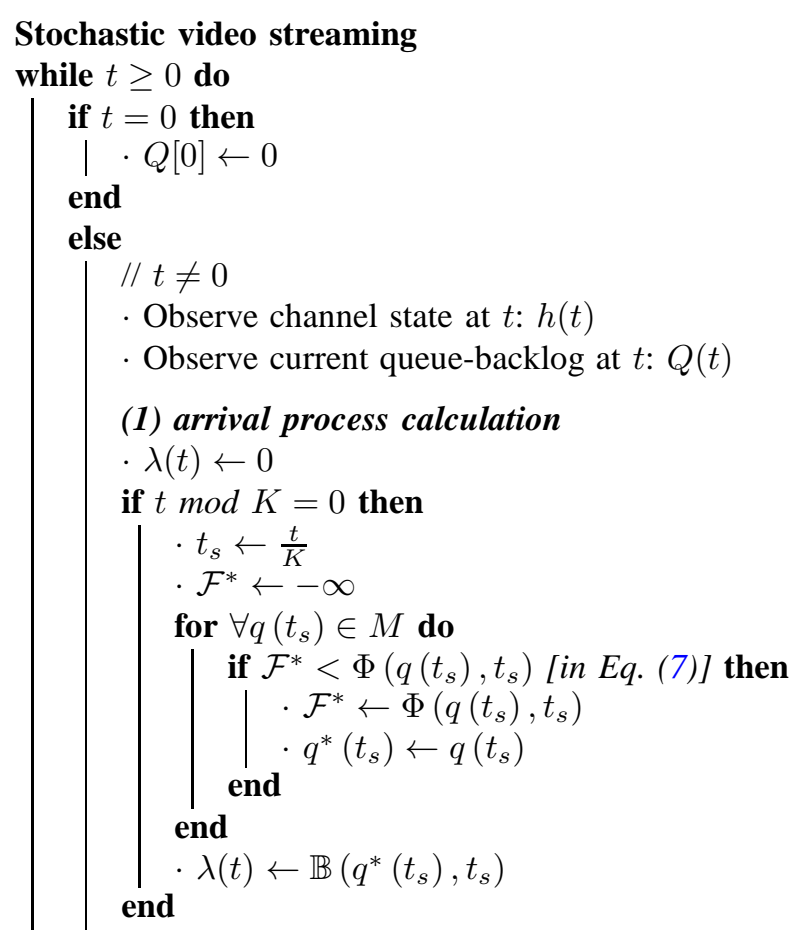

(2) departure process calculation

$$
\cdot \mu(t) \leftarrow \mathrm{BW} \cdot \log _{2}\left(1+\frac{P_{\mathrm{mW}}^{\mathrm{Tx}} \cdot\|h(t)\|^{2}}{N_{\mathrm{mW}}}\right)
$$

\section{(3) queue update}

\rceil$_{\text {end }} \cdot Q(t+1) \leftarrow \max \{Q(t)+\lambda(t)-\mu(t), 0\}$

Algorithm 1: Pseudo-code for stochastic streaming [3]

$$
q^{*}\left(t_{s}\right) \leftarrow \arg \max _{q\left(t_{s}\right) \in M} \Phi\left(q\left(t_{s}\right), t_{s}\right)
$$

where

$$
\Phi\left(q\left(t_{s}\right), t_{s}\right) \triangleq \mathbb{P}\left(q\left(t_{s}\right), t_{s}\right)-V \cdot \mathbb{B}\left(q\left(t_{s}\right), t_{s}\right) \cdot Q(t)
$$

where $M$ is the set of possible quality modes, $V$ is a tradeoff parameter between quality maximization and queue stability (if this $V$ is small, the optimization framework put more priority on quality maximization, and vice versa), and $\mathbb{B}\left(q\left(t_{s}\right), t_{s}\right)$ is the size (i.e., bitrate) of the stream in stream time $t_{s}$ when the quality mode is $q\left(t_{s}\right)$.

Since the placement of streams constitutes the arrival process of the queue, $\lambda(t)$ can be denoted as follows when the 


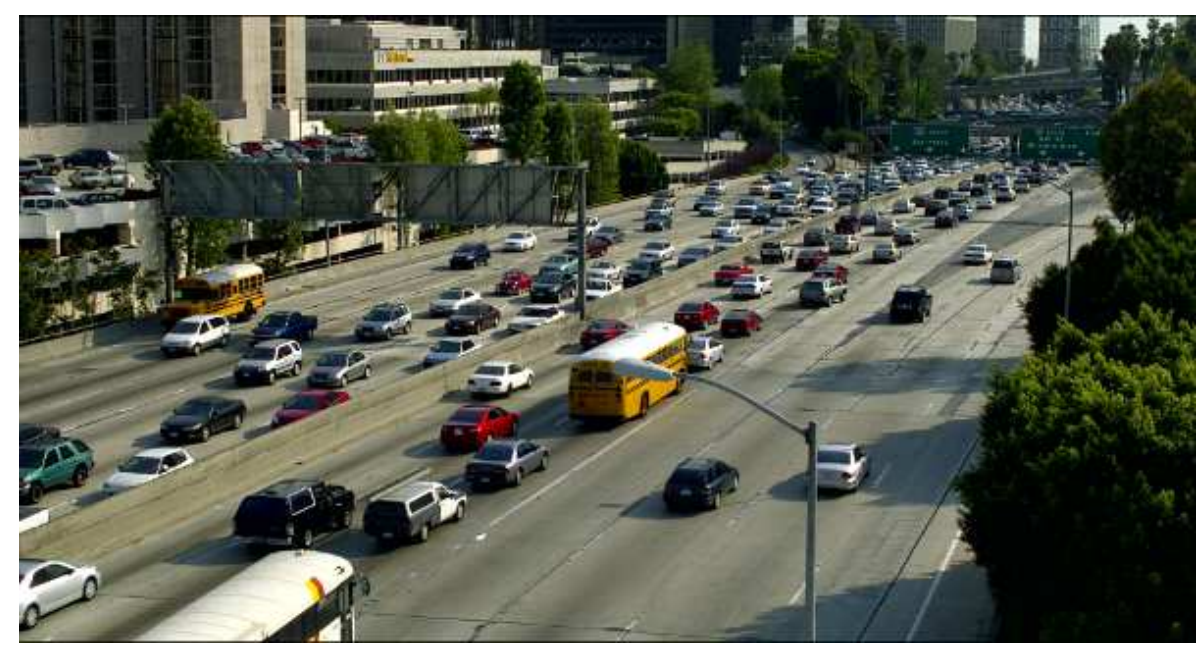

Fig. 2. A sample $4 \mathrm{~K}$ UHD video frame

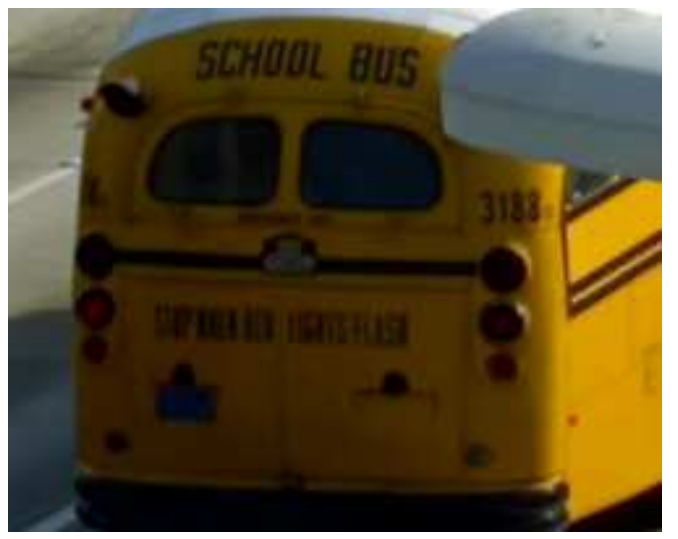

(a) QP: 22

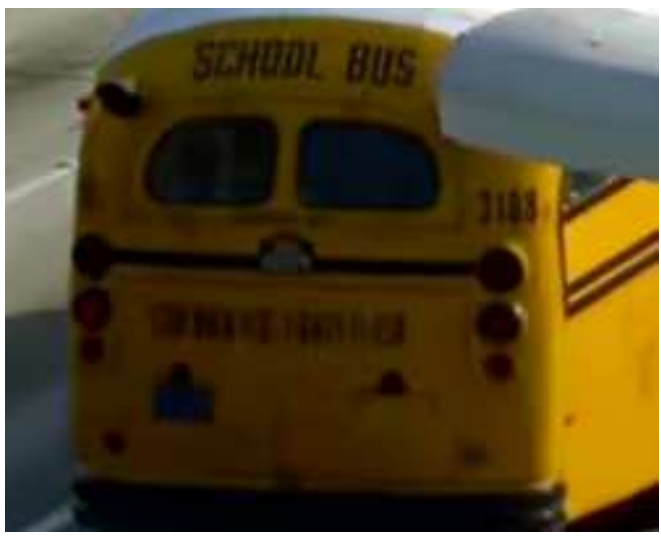

(c) QP: 32

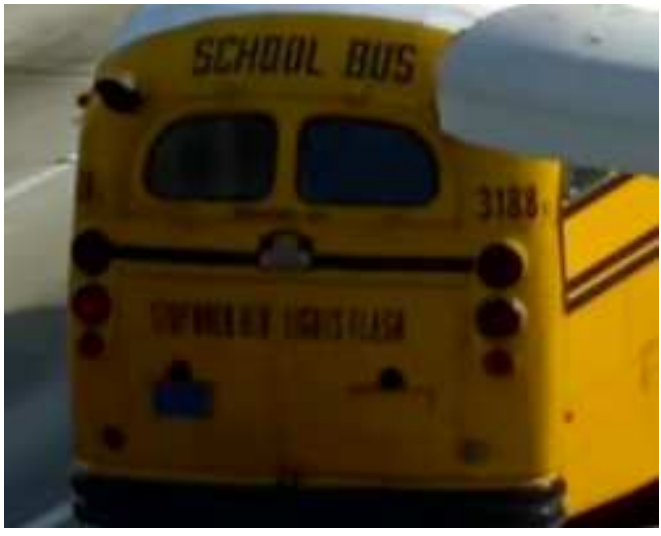

(b) QP: 27

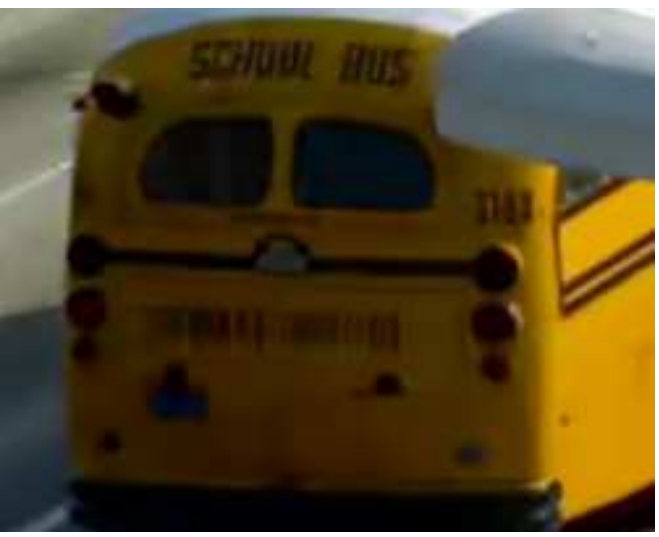

(d) QP: 37

Fig. 3. Test video sequences \#1: School bus

optimal $q^{*}\left(t_{s}\right)$ is determined using Eq. (6).

$$
\lambda(t)= \begin{cases}\mathbb{B}\left(q^{*}\left(t_{s}\right), t_{s}\right), & t_{s} \bmod K=0 \\ 0, & t_{s} \bmod K \neq 0\end{cases}
$$

2) Departure Process (Transmission of Bits): As illustrated in Fig. 1, the departure process in the given system is the 


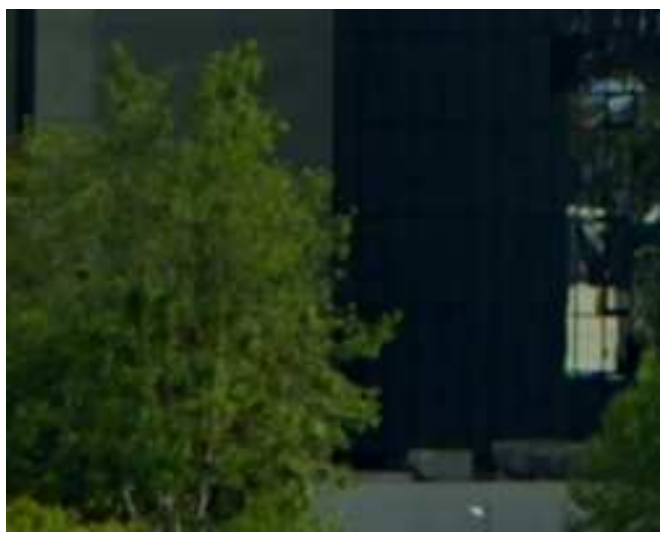

(a) QP: 22

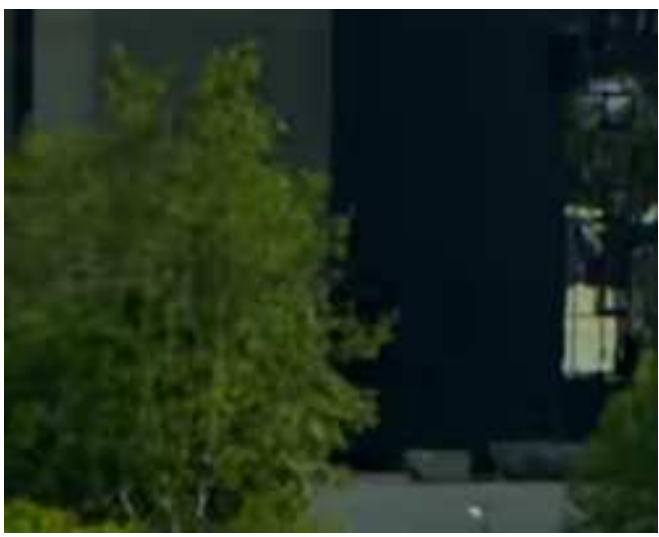

(c) QP: 32

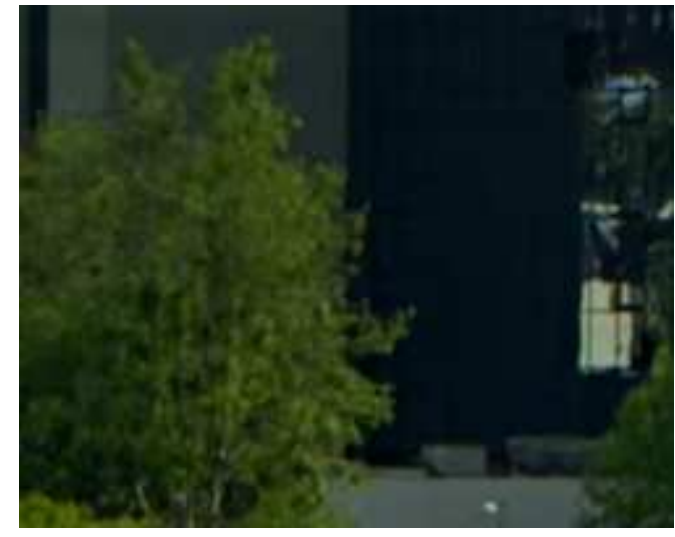

(b) QP: 27

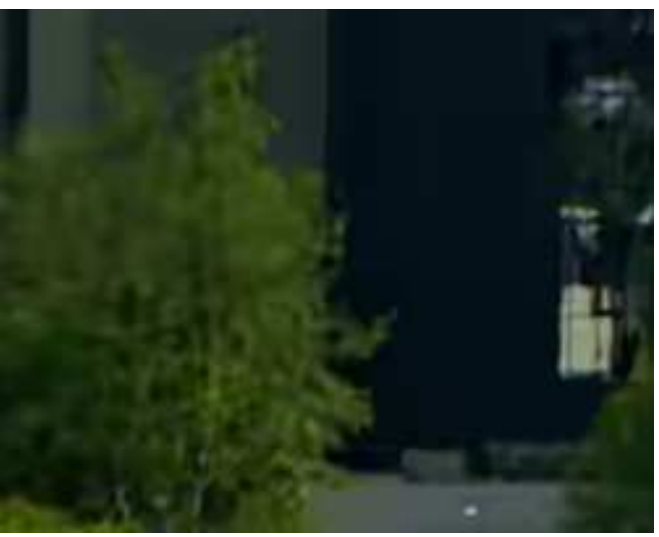

(d) QP: 37

Fig. 4. Test video sequences \#2: Trees and buildings

transmission of bits. We can transmit bits as much as the wireless channel allows. In conventional wireless standards (e.g., IEEE 802.11, IEEE 802.15, or 3GPP LTE), modulation and coding scheme (MCS) sets are defined and the corresponding wireless links can transmit bits based on the defined MCS rules. In this paper, we are not considering specific standard protocols. Therefore, theoretical Shannon's capacity equation is considered and it can be calculated as follows [9]:

$$
\mu(t)=\mathrm{BW} \cdot \log _{2}\left(1+\frac{P_{\mathrm{mW}}^{\mathrm{Tx}} \cdot\|h(t)\|^{2}}{N_{\mathrm{mW}}}\right)
$$

where $P_{\mathrm{mW}}^{\mathrm{Tx}}$ stands for the power transmitted by a transmitter to its associated receiver in a milli-Watt scale, $h(t)$ stands for the channel gain from the transmitter to its associated receiver at time $t, N_{\mathrm{mW}}$ is a background noise in the system in a milliWatt scale, and BW stands for the channel bandwidth of the system. In (9), the $N_{\mathrm{mW}}$ is assumed to be 1 in this paper.

Finally, the pseudo-code of the proposed stochastic video streaming in [3], [4] is as presented in Algorithm 1.

\section{FEASIBILITY STUDY}

\section{A. Test Sequence Generation}

The computing environments and original video information for video trace generation are as follows:

- Resolution: 3840-by-2048 (for 4K UHD video)

- Frame rate: $30 \mathrm{fps}$ (30 frames per second)

- Bit depth: 8 bits

- Test sequence name: Traffic (for video standard testing)

- Profile name: Main

- Intra Period: 32

- GOP size: 8

- Four different video qualities with QP (quantization Parameters): 22, 27, 32, and 37

- Encoder: HM version 15.0 (HEVC standard reference codes)

- PC: Intel i7 CPU, Windows7 64bit OS

\section{B. $4 K$ UHD Video Traces}

With the computing and parameter settings as presented in Section III-A, 4K UHD test video traces are generated and the 
representative sample full video frame is as presented in Fig. 2. Two parts of the full video frame are as presented in Fig. 3 and Fig. 4. For each part, the compression results are presented in each Fig. 3 and Fig. 4. As explained in Section III-A, we have four different quality levels, i.e.,

$$
M=\{\mathrm{QP}=22, \mathrm{QP}=27, \mathrm{QP}=32, \mathrm{QP}=37\}
$$

and $q\left(t_{s}\right) \in M$. For each quality level, visual compression results are presented in Fig. 3 and Fig. 4 for the given two difference parts in the full video frame.

In addition, the PSNR and Bitrate are measured in each quality level for 10 sample streams; and the corresponding measured results are presented in Table I.

\section{Results}

With the given numerical information in Section III-B, the performance of stochastic streaming is evaluated and compared with following two streaming algorithms:

- Queue-independent streaming with maximum quality (QP is set to 22), named to [QP22]

- Queue-independent streaming with minimum quality (QP is set to 37), named to [QP37]

The simulation is with following two criteria: (i) various $K$ setting (refer to Section III-C1) and (ii) various $V$ setting (refer to Section III-C2).

1) Simulation with various $K$ settings: For the simulation with various $K$, we consider two cases, i.e., (i) $K=10$ and (ii) $K=1$. We run the simulation for 3000 unit times. In addition, the transmit power and channel bandwidth are assumed to be $5 \mathrm{dBm}$ and $1 \mathrm{MHz}$, respectively. The simulation results with $K=10$ and $K=1$ are plotted as shown in Fig. 5(a) and Fig. 5(b), respectively.

As shown in both Fig. 5(a) $(K=10)$ and Fig. 5(b) $(K=1)$, the queue/buffer diverges if we place streams with the highest quality (i.e., $\mathrm{QP}=22$ ) as shown in the [QP22] plots. Fig. 5(a) shows that the queue/buffer backlog size is always zero if we place streams with the lowest quality (i.e., $\mathrm{QP}=37$ ). If $K=1$ (i.e., Fig. 5(b)), the [QP37] is starting to increase because the placement of streams is frequently occurring. As shown in both Fig. 5(a) $(K=10)$ and Fig. 5(b) $(K=1)$, the proposed stochastic streaming starts to show convergence trends when the unit time is approximately 800 . Even though both shows convergence trends, the case with $K=10$ is more stable because it places the streams sparser than the placement of streams with $K=1$.

2) Simulation with various $V$ settings: For the simulation with various $V$, we consider two cases, i.e., (i) $V=10^{-16}$ and (ii) $V=5 \times 10^{-16}$. We run the simulation for 3000 unit times and we also assume that $K=1$. In addition, the transmit power and channel bandwidth are assumed to be $5 \mathrm{dBm}$ and $1 \mathrm{MHz}$, respectively. The simulation results with $V=10^{-16}$ and $V=5 \times 10^{-16}$ are plotted as shown in Fig. 6 .

As shown in Fig. 6, the queue/buffer also diverges if we place streams with the highest quality (i.e., $\mathrm{QP}=22$ ) as shown in the [QP22] plots. As presented in Fig. 6, both the stochastic streaming algorithms with $V=10^{-16}$ and
TABLE I

VIDEO TRACE INFORMATION

\begin{tabular}{|c|c|c|c|}
\hline Stream \# & $\begin{array}{r}\text { Quality \# } \\
q\left(t_{s}\right)\end{array}$ & $\begin{array}{r}\operatorname{PSNR}(\mathrm{dB}) \\
\mathbb{P}\left(q\left(t_{s}\right), t_{s}\right)\end{array}$ & $\begin{array}{r}\text { Bitrate (Kbps) } \\
\mathbb{B}\left(q\left(t_{s}\right), t_{s}\right)\end{array}$ \\
\hline 1 & 1 (QP: 22) & 41.64 & 26496 \\
\hline 1 & 2 (QP: 27) & 39.11 & 10658 \\
\hline 1 & 3 (QP: 32) & 36.61 & 5073 \\
\hline 1 & 4 (QP: 37) & 34.00 & 2621 \\
\hline 2 & 1 (QP: 22) & 41.64 & 26811 \\
\hline 2 & 2 (QP: 27) & 39.07 & 10811 \\
\hline 2 & 3 (QP: 32) & 36.56 & 5128 \\
\hline 2 & 4 (QP: 37) & 33.97 & 2650 \\
\hline 3 & 1 (QP: 22) & 41.60 & 27888 \\
\hline 3 & 2 (QP: 27) & 39.00 & 11279 \\
\hline 3 & 3 (QP: 32) & 36.48 & 5320 \\
\hline 3 & 4 (QP: 37) & 33.91 & 2721 \\
\hline 4 & $1(\mathrm{QP}: 22)$ & 41.61 & 27145 \\
\hline 4 & 2 (QP: 27$)$ & 39.05 & 10958 \\
\hline 4 & 3 (QP: 32) & 36.53 & 5193 \\
\hline 4 & 4 (QP: 37) & 33.94 & 2679 \\
\hline 5 & $1(\mathrm{QP}: 22)$ & 41.63 & 26535 \\
\hline 5 & 2 (QP: 27) & 39.08 & 10710 \\
\hline 5 & 3 (QP: 32) & 36.57 & 5095 \\
\hline 5 & 4 (QP: 37) & 33.98 & 2636 \\
\hline 6 & $1(\mathrm{QP}: 22)$ & 41.60 & 27630 \\
\hline 6 & 2 (QP: 27) & 39.02 & 11130 \\
\hline 6 & 3 (QP: 32) & 36.51 & 5263 \\
\hline 6 & 4 (QP: 37) & 33.94 & 2703 \\
\hline 7 & 1 (QP: 22) & 41.61 & 27766 \\
\hline 7 & 2 (QP: 27) & 39.01 & 11237 \\
\hline 7 & 3 (QP: 32) & 36.49 & 5303 \\
\hline 7 & 4 (QP: 37) & 33.91 & 2714 \\
\hline 8 & 1 (QP: 22) & 41.63 & 26689 \\
\hline 8 & 2 (QP: 27) & 39.10 & 10765 \\
\hline 8 & 3 (QP: 32) & 36.59 & 5118 \\
\hline 8 & 4 (QP: 37) & 34.00 & 2641 \\
\hline 9 & 1 (QP: 22) & 41.62 & 27083 \\
\hline 9 & 2 (QP: 27) & 39.06 & 10902 \\
\hline 9 & 3 (QP: 32) & 36.56 & 5181 \\
\hline 9 & 4 (QP: 37) & 33.97 & 2678 \\
\hline 10 & 1 (QP: 22) & 41.60 & 28006 \\
\hline 10 & 2 (QP: 27) & 39.00 & 11378 \\
\hline 10 & 3 (QP: 32) & 36.47 & 5364 \\
\hline 10 & 4 (QP: 37) & 33.89 & 2735 \\
\hline
\end{tabular}

$V=5 \times 10^{-16}$ also present convergence trends; and the algorithm with $V=10^{-16}$ shows the higher queue-backlog sizes because the algorithm with lower $V$ pursues time-average quality maximization rather than queue stability.

\section{CONCLUSiOnS AND Future WORK}

This paper shows the feasibility study results of stochastic streaming algorithms with $4 \mathrm{~K}$ ultra-high-definition (UHD) video traces. In literatures, various stochastic streaming algorithms have been proposed that maximize time-average streaming quality subject to queue stability under the consideration of queue-backlog size. The performance improvements 


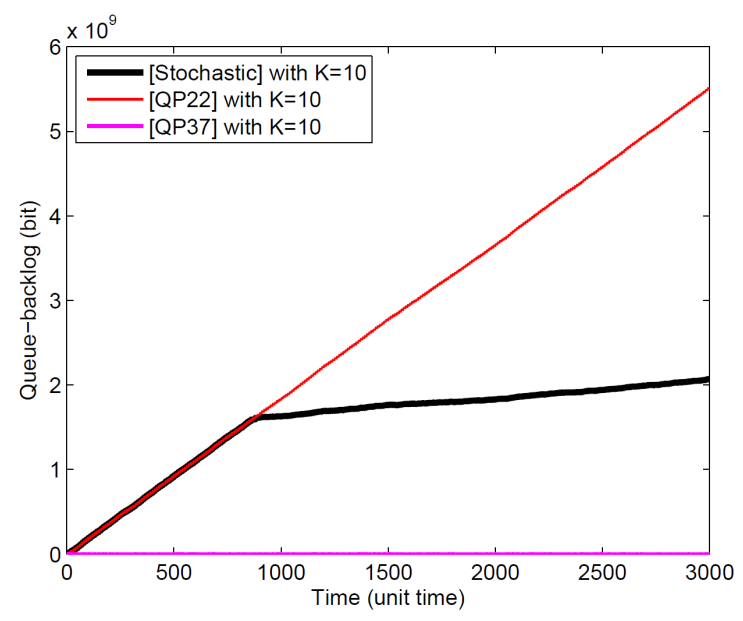

(a) $K=10$

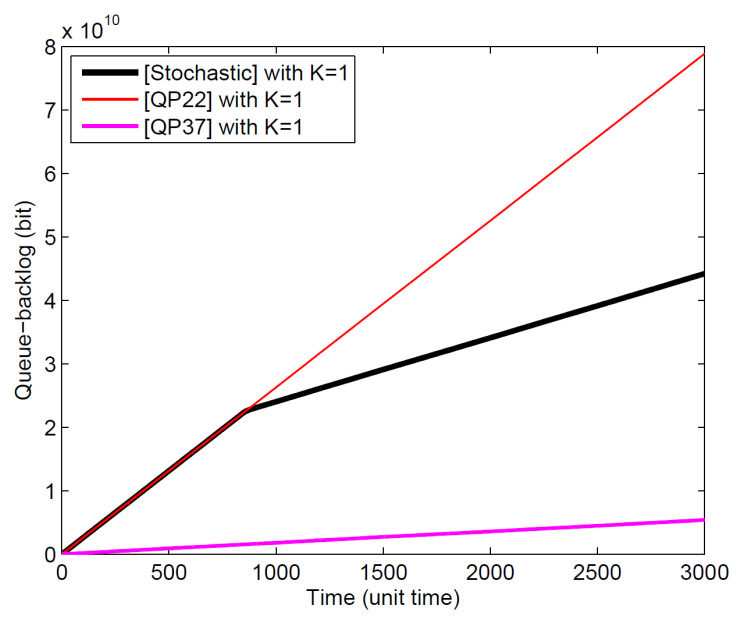

(b) $K=1$

Fig. 5. Simulation results with various $K\left(V=10^{-16}\right)$

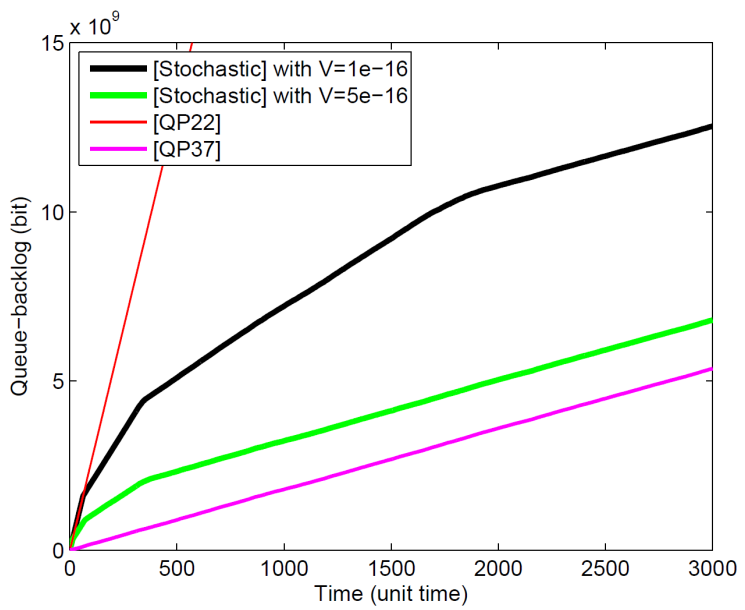

Fig. 6. Simulation results with various $V(K=1)$

with the stochastic video streaming algorithms were verified with traditional MPEG test sequences in previous work; however there are no research results with up-to-date $4 \mathrm{~K}$ UHD video traces. Thus, this paper verifies the performance of the stochastic streaming algorithms with $4 \mathrm{~K}$ UHD video traces; and shows that the stochastic algorithms perform better than queue-independent algorithms.

\section{ACKNOWLEDGEMENT}

This research was supported by Basic Science Research Program through the National Research Foundation of Korea(NRF) funded by the Ministry of Science, ICT \& Future Planning (NRF-2015R1C1A1A02037743). E.-S. Ryu is a corresponding author of this paper.

\section{REFERENCES}

[1] Cisco, "Cisco Visual Networking Index: Forecast and Methodology 2012 2017," Cisco White Paper, 2013.

[2] N. Golrezaei, A. F. Molisch, A. G. Dimakis, and G. Caire, "Femtocaching and Device-to-Device Collaboration: A New Architecture for Wireless Video Distribution," IEEE Communications Magazine, vol. 51, no. 4, pp. 142-149, April 2013.

[3] J. Kim, G. Caire, and A.F. Molisch, "Quality-Aware Streaming and Scheduling for Device-to-Device Video Delivery," IEEE/ACM Transactions on Networking, Published Online, July 2015., DOI: 10.1109/TNET.2015.2452272

[4] J. Kim, A.F. Molisch, and G. Caire, "Max-Weight Scheduling and Quality-Aware Streaming for Device-to-Device Video Delivery," arXiv:1406.4917, June 2014.

[5] J. Kim, F. Meng, P. Chen, H.E. Egilmez, D. Bethanabhotla, A.F. Molisch, M.J. Neely, G. Caire, and A. Ortega, "Demo: Adaptive Video Streaming for Device-to-Device Mobile Platforms," in Proceedings of the ACM International Conference on Mobile Computing and Networking (MobiCom'13), Miami, FL, USA, September 2013.

[6] D. Bethanabhotla, G. Caire, and M. J. Neely, "Joint Transmission Scheduling and Congestion Control for Adaptive Streaming in Wireless Device-to-Device Networks" in Proceedings of the Asilomar Conference on Signals, Systems, and Computers (Asilomar'12), Pacific Grove, CA, USA, November 2012.

[7] D. Bethanabhotla, G. Caire, and M. J. Neely, "Adaptive Video Streaming for Wireless Networks With Multiple Users and Helpers," IEEE Transactions on Communications, vol. 63, no. 1, pp. 268-285, January 2015.

[8] D. Bethanabhotla, G. Caire, abd M. J. Neely, "Utility Optimal Scheduling and Admission Control for Adaptive Video Streaming in Small Cell Betworks," in Proceedings of the IEEE International Symposium on Information Theory (ISIT'13), Istanbul, Turkey, July 2013.

[9] A.F. Molisch, Wireless Communications, 2nd Edition, IEEE-Wiley, February 2011.

[10] M. J. Neely, Stochastic Network Optimization with Application to Communication and Queueing Systems, Morgan \& Claypool, 2010.

[11] J. Kim, Y. Tian, S. Mangold, and A.F. Molisch, "Joint Scalable Coding and Routing for $60 \mathrm{GHz}$ Real-Time Live HD Video Streaming Applications," IEEE Transactions on Broadcasting, vol. 59, no. 3, pp. 500-512, September 2013. 